\title{
La mediación filosófica en el diálogo
} entre ciencia y teología: un análisis del concepto de consonancia (Philosophical intervention in the dialogue between science and theology: an analysis of the concept of consonance)

\author{
OSCAR BELTRÁN \\ Pontificia Universidad Católica Argentina \\ oscarbeltran@uca.edu.ar \\ ORCID: 0000-0001-9689-9409
}

Resumen. En las últimas décadas se ha suscitado un intenso debate acerca de la relación entre ciencia y teología. A comienzos de 1980 Ernan McMullin introdujo la noción de "consonancia" definida como la confluencia espontánea de las conclusiones elaboradas por las ciencias de la naturaleza y la teología acerca de determinados rasgos o propiedades del universo. En este trabajo se intentará, ante todo, indagar con detenimiento acerca del sentido que se le ha atribuido a esta relación. Así se examinarán las posibles implicaciones derivadas de los casos puntuales de consonancia; se considerará la conexión entre consonancia y verdad (así como, simétricamente, la que podría establecerse entre "disonancia" y falsedad); se avanzará en el planteo de un diálogo basado en diversas actitudes respecto a la consonancia (rechazo, indiferencia, aceptación y promoción); se evaluará la tesis que interpreta la consonancia como una construcción cultural o un a priori asumido en determinados contextos; finalmente, se 
hará mención de ciertas variantes de este concepto, como las de consonancia fuerte y débil, o consonancia hipotética. Luego se expondrán los supuestos sobre los que descansa la idea de consonancia: la unidad e inteligibilidad de lo real, la diversidad de los objetos formales y el realismo del conocimiento. Todos estos supuestos representan un testimonio cabal de la indispensable mediación que le cabe ejercer a la filosofía, desde su carácter sapiencial, para cualquier tipo de intercambio que se auspicie entre la ciencia y la teología. Y es solo desde ellos que se inviste de autoridad la crítica que se pueda hacer respecto de esta cuestión. Finalmente se propone una serie de planteos críticos acerca de la noción de consonancia. Se subrayará, desde lo positivo, la oportunidad que ofrece para avanzar hacia una mayor integración de las disciplinas y promover, al mismo tiempo, la misión ordenadora que le corresponde a la sabiduría filosófica. En cuanto a lo negativo, se hará notar la confusión entre religión y teología, la tendencia a una suerte de naturalismo epistémico de la teología, la elaboración precaria de la idea de realismo científico y la pretensión de una excesiva injerencia entre disciplinas.

Palabras clave: diálogo; consonancia; ciencia; teología; filosofía.

Abstract. In the last decades, a strong debate on the relationship between science and theology has taken place. In the early 1980, Ernan McMullin introduced the notion of "consonance", defined as the spontaneous convergence of the conclusions presented by the natural sciences and theology on certain features or properties of the universe. In this paper, I shall try, first and foremost, to carefully look into the meaning that has been attributed to this relationship. Thus, I shall examine the possible implications derived from specific cases of consonance; I shall consider the connection between consonance and truth (as well as, symmetrically, the one that could be established between "dissonance" and falseness); I shall expand on the proposal for a dialogue based on different attitudes regarding consonance (rejection, indifference, acceptance and promotion); I shall evaluate the thesis that understands consonance as a cultural construction or an a priori accepted in certain contexts; finally, I shall mention certain variations of this concept, such as strong and weak consonance, or hypothetical consonance. Then, I shall explain the assumptions on which the idea of consonance rests: the unity and intelligibility of reality, the diversity of formal objects and the realism of knowledge. All these assumptions are comprehensive proof of the indispensable intervention of philosophy, as wisdom, in any kind of exchange that occurs between science and theology. And it is only from them that criticism regarding this issue is vested authority. Finally, I suggest a series of critical proposals on the notion of consonance. I shall stress, on the positive side, the opportunity that it offers to move towards a greater disciplinary integration and foster, at the same time, the ordering mission of philosophical wisdom. On the negative side, I shall point out the confusion between religion and theology, the tendency towards a kind of epistemic naturalism of theology, the uncertain creation of the idea of scientific realism and the aspiration to an excessive interference among disciplines.

Keywords: dialogue; consonance; science; theology; philosophy. 
[...] nada es más consonante con la razón que aquello que ha de creerse a Dios en todo cuanto dice y se testimonia.

Tomás de Aquino In IV Sent. 9, 1, 3d ad 2m

\section{Introducción}

El querido y recordado Mariano Artigas, en uno de sus trabajos más logrados, decía: "por primera vez en la historia disponemos de una cosmovisión científica que proporciona una imagen rigurosa y unificada del mundo, porque incluye todos los niveles naturales (el microfísico y el macrofísico, incluyendo el biológico) y sus relaciones mutuas" (Artigas 2000, 101). La feliz combinación de un alcance cada vez más ambicioso y un rigor cada vez más estricto han convertido a la ciencia en un interlocutor natural de los saberes sapienciales. Tanto desde lo que podrían denominarse "temáticas fronterizas" como desde el impacto personal que ejercen estas especulaciones en el ánimo de los científicos, desde hace un tiempo parece llegada la hora de acometer, de manera sistemática y profunda, el diálogo entre la filosofía, la teología y la ciencia tantas veces empañado por los conflictos, y sobre el cual la comunidad intelectual y las instituciones religiosas han puesto ya su esperanza.

Podemos decir que estamos en presencia de un fenómeno cultural inédito. No es atrevido considerarlo como un auténtico signo de los tiempos que puede constituirse en un tema de reflexión en sí mismo. San Juan Pablo II supo ver en su momento la importancia apremiante de este fenómeno, y a causa de su desvelo profético nos ha dejado un generoso magisterio acerca de él (Papanicolau 2003). La pieza más destacada, después de la Fides et Ratio, es para muchos la Carta que dirigió en 1987 al entonces titular del Observatorio Vaticano, George Coyne (Juan Pablo II 1989). Por entonces estaban en germen varias de las iniciativas que la Iglesia y otras organizaciones confesionales promovieron para fomentar el diálogo. Por eso el Papa expresaba allí que el modo preciso de la comunicación entre ciencia y teología “debe dejarse para el futuro". A casi tres décadas de este pronunciamiento parece razonable pensar en un intento por dar una forma más o menos estable a ese intercambio. 


\section{Un caso testigo: la idea de consonancia}

Son bien conocidas las diferentes tipologías que se han propuesto para describir los modos de relación entre la ciencia y la teología. Con más o menos matices, la mayoría de ellas puede considerarse una variación del esquema cuatripartito de Ian Barbour. En general se tiende a pensar que las categorías de Barbour representan más bien estereotipos a la manera de los colores primarios, de cuya combinación pueden obtenerse múltiples modalidades que se postulan como esquemas más exactos de representación de aquella relación. Incluso se afianza la suposición de que ninguna de esas combinaciones puede dar cuenta de todas las instancias en que se vinculan la ciencia y la teología, de modo que se deba echar mano a diversas opciones según el caso.

En esta línea me ha llamado la atención una propuesta del sacerdote, físico y filósofo de la ciencia Ernan McMullin, planteada una década antes de publicarse las Conferencias Gifford en las que Barbour expone su taxonomía, y que ha sido acogida luego por otros eminentes investigadores, como Ted Peters (1998) y John Polkinghorne, quien afirma que "The search for consonance is the basis of my own approach to the question of interrelationship." (1996, 7). Se trata del término consonance (consonancia), con el cual se intenta, al menos en principio, definir un vínculo de convergencia espontánea entre las ciencias y la teología. La consonancia se dará cada vez que, siguiendo su propio derrotero, la investigación científica y la reflexión teológica confluyan en su concepción acerca de algún tema. El ejemplo típico, en tal sentido, es el de la teoría del Big Bang y la idea de un comienzo absoluto del universo, en directa concordancia con la noción teológica de creación ex nihilo. A continuación intentaré presentar con mayor amplitud las líneas principales de esta discusión seguidas de una apreciación crítica.

\section{La propuesta de McMullin}

En setiembre de 1979 tuvo lugar en Oxford un simposio internacional sobre el tema Las ciencias y la teología en el siglo XX. En su contribución a este 
encuentro McMullin introdujo una nueva figura para la caracterización del diálogo entre teología y ciencia, que denominó consonancia (McMullin 1981). En la primera parte de su trabajo examina dos modelos de confrontación entre la fe y la ciencia. El primero de ellos, designado como principio de relevancia, está inspirado en San Agustín. Según el Doctor de Hipona, cada vez que un pasaje bíblico contradice una verdad científica debidamente probada debe tomárselo metafóricamente. No obstante, todo lo que se diga en nombre de la ciencia que entre en conflicto directo con la fe católica debe ser rechazado como falso. El criterio agustiniano resulta estrecho porque exige a la ciencia natural que demuestre rigurosamente sus conclusiones, lo cual es imposible en casi todos los casos. Cuando la conclusión sea probable, prevalece la versión bíblica (McMullin 1981, 27). El segundo, al que llama principio de neutralidad, está ejemplificado en la obra de Galileo, más concretamente en su célebre carta a la Gran Duquesa Cristina de Lorena. Hay en el pensamiento del Pisano una ambivalencia: por momentos adhiere al criterio fuerte de San Agustín (sólo prevalece la ciencia sobre la Biblia cuando la proposición esté estrictamente demostrada), y por momentos introduce una distinción más sutil: entre aquellas afirmaciones que definitivamente escapan a la certeza humana, y aquellas que puede esperarse que sean demostradas en el futuro, de manera que solo acerca de las primeras debe preferirse la lectura literal de la Biblia. Más aún, en otro pasaje Galileo propone el criterio según el cual el texto sagrado debe tenerse en cuenta puntualmente cuando se trate de asuntos referidos al mensaje de salvación. En todo lo demás habrá que preferir la palabra de la ciencia. De esta manera ya no cuenta si la teoría copernicana está demostrada o no. Simplemente se trata de algo ajeno al mensaje de salvación, y por eso la teología queda fuera de la discusión. Vale recordar que en su carta a Foscarini, el Cardenal Bellarmino rechaza este criterio ya que incluso aquellas verdades que no son materia de fe por lo que en ellas se dice, lo son por parte del que lo dice, es decir, el Espíritu Santo.

A partir de la encíclica Providentissimus Deus de León XIII (1893) se ha impuesto este último principio, mientras la regla agustiniana de la relevancia fue adoptada por las diferentes expresiones del fundamentalismo. 
Pero todavía queda una cuestión pendiente de resolver. No hay duda de que ciertas afirmaciones de la doctrina cristiana sobre el hombre (libertad, responsabilidad) tienen injerencia directa en ciencias como la psicología o la sociología. La pregunta es si la Biblia y el dogma influyen, en algún caso, respecto al contenido de la cosmología, o sea si la fe limita u orienta, según el caso, lo que las ciencias naturales puedan decir acerca del mundo:

The question of how science and religion should interrelate is not primarily either a scientific or a theological one. Nor is it primarily historical or sociological, though one will surely want to know much about the historical relations of the two, as well as about the sociological issues these relations raise, before coming to any conclusions. The question is primarily an epistemological one about how two different sorts of claim to knowledge are to be related. In discussing it, it is important to be sensitive to the complexities of the properly scientific and properly theological issues involved. It is also important to be aware of the historical records and the relevant sociological observations and of what may be learned from them. But in the end, the question itself is a characteristically epistemological one, one of great complexity." [...] "This is not to say that the philosopher is necessarily the person best qualified to discuss it. The context will indicate the degree of expertise required in these other areas, and it may well be that a scientist or historian sensitive to the issues may contribute most to some aspects of the topic. But when they do, they are contributing to a problem which is basically one about the nature of knowledge. And this is not a matter of science nor of theology, let alone of history or of sociology. (McMullin 1981, 25-26).

Por otra parte, la ciencia ha profundizado sus conocimientos en la línea adyacente a la temática metafísica y religiosa.

Diversos hechos han impulsado un examen profundo de los supuestos de la nueva cosmología. McMullin menciona, en primer lugar, el discurso de Pío XII a la Academia Pontifica de Ciencias de 1951, en el que destacó el valor de los nuevos hallazgos de la cosmología como un notable refuerzo de la certidumbre de las verdades filosóficas tradicionales, en particular la de la existencia de Dios. En este punto va más allá de la afirmación de una conciliación entre ciencia y fe, para hablar de un aporte de evidencia de las teorías científicas a favor de la existencia de Dios. Pero además surgieron 
voces discordantes con la teoría estándar del origen del universo, como la de Fred Hoyle, para quien la hipótesis del Big Bang está demasiado cercana a la idea cristiana de creación, y justamente por eso debe ser combatida. (McMullin 1981, 34)

Según sostiene McMullin, la ciencia se ve obligada a rechazar metodológicamente la idea de un comienzo absoluto o de una singularidad, pero eso no significa que no pueda haber un comienzo absoluto:

Scientists ought not assume that the Big Bang had no antecedent; they ought to do whatever they can to establish a law-like succession. But this is not to say that there must be an antecedent, that the success of these principles demonstrates that an absolute beginning is imposible. This is a metaphysical claim, and it would require more on its behalf than a mere inductive appeal to the success up to this point of the genetic and conservation principles. (McMullin 1981, 38)

A juicio de este autor, pueden establecerse a esta altura tres conclusiones: "primero, que el modelo del Big Bang no da derecho a inferir un comienzo absoluto del tiempo; segundo, que no hay nada inadmisible desde el punto de vista científico o filosófico acerca de la suposición de que un comienzo absoluto pueda haber ocurrido (aunque en un terreno metodológico tal comienzo debería concederse únicamente si todas las clases familiares de continuidad pudiesen ser excluidas); y, tercero, que si un comienzo cósmico absoluto ocurrió, puede resultar algo tal como el evento-horizonte descripto en la teoría del Big Bang” (McMullin 1981, 40). Cabe preguntarse si la afirmación de que el universo tuvo un comienzo temporal refuerza la idea de un Dios creador. Santo Tomás pensaba que sí, aunque tenía en claro que un mundo eterno exige igualmente la intervención divina. En todo caso, "lo que uno podría, no obstante, decir sin reparos es que si el universo comenzó en el tiempo mediante el acto de un Creador, desde nuestra perspectiva privilegiada su aspecto sería tal como el Big Bang del que los cosmólogos están hablando actualmente. Lo que no se puede decir es, primero, que la doctrina cristiana de la creación "respalda” (support) el modelo del Big Bang, o segundo, que el modelo del Big Bang “respalda” la teoría cristiana de la creación” (McMullin 1981, 40). 
Luego de discutir el ejemplo del principio antrópico, McMullin observa que en la teología actual se tiende a considerar en términos neutrales la relación entre cosmología y fe bíblica, dado que la intención del texto sagrado es ajena a la información científica, y sus géneros literarios están ambientados al modo de comprensión de los hombres de su época. Pero en el caso del hombre existen puntos de contacto insoslayables, sobre todo en relación a la libertad y a la estructura alma-cuerpo.

En varios de sus trabajos, McMullin ha expresado su rechazo hacia ciertos planteos de una "teología natural" que propone, por diversos caminos, una concepción indebidamente inmanentista de la causalidad divina. Tal es para él la situación en el caso del debate acerca del concepto de "creación evolutiva”. A su entender, la propuesta de Alvin Plantinga de una creación especial que supla la falta de eslabones; o la presencia de un élan vital a la manera de Bergson, reinterpretado en clave cristiana por Teilhard de Chardin y sistematizado en la llamada teología del proceso, son enfoques deficientes tanto en sentido metafísico como epistemológico.

Lo primero, en virtud de la univocación de la causalidad en sentido puramente físico-temporal, sin tener en cuenta la esencial trascendencia del obrar divino. En efecto, el acto creador de Dios procede de Él sin distinguirse de Su plena Unidad y Simplicidad de manera que, visto desde Dios mismo, no es otra cosa que participación del ser. Pero es en la recepción de ese acto (término inadecuado, puesto que el crear se define ex nihilo subiecti en cuanto donación de todo ser) que se despliega la variedad de lo existente y la compleja trama de determinación, azar y contingencia que la mirada científica registra.

Lo segundo, porque compromete en demasía la autonomía de las ciencias, acuñando una especie de teología física que parece subalternarse a la ciencia natural a la manera en que la misma física lo hace respecto a la matemática. Es decir, sería un vínculo más bien de integración que de diálogo. Además, este afán conciliador suele ir de la mano de un conocimiento desactualizado de las teorías científicas, o de la presunción de que los agujeros que la ciencia deja expuestos hoy no podrán ser rellenados en el futuro. El estudio recién citado señala la teoría de las razones seminales de San Agustín como una opción que deja a salvo la trascendencia divina y 
resulta, a entender de McMullin, plenamente consonante con los modelos científicos evolutivos (Barzaghi y Corcó 2015, 520-521).

En lugar de plantear un saber teológico con implicaciones que afecten directamente el contenido de la ciencia, se puede asumir dicho saber como una instancia que ayude a interpretar el sentido de las teorías científicas en un contexto de visión del mundo: "Esto sería tomar la teología no como una fuente autónoma de implicación lógica capaz de afectar la apreciación científica de una teoría, sino como un elemento en la construcción de una visión más amplia del mundo" Y aquí surge la propuesta clave:

El objetivo podría ser la consonancia más que la implicación directa [...] El cristiano no puede separar su ciencia de su teología como si fueran en principio incapaces de interrelación. Por otra parte, ha aprendido a desconfiar de los caminos más simples de un punto al otro. Debe aspirar a una suerte de coherencia en la visión del mundo, una coherencia a la cual deben contribuir la ciencia y la teología, y ciertamente muchas otras formas de construcción humana, como la historia, la política y la literatura. Puede, de hecho, debe esforzarse por hacer consonantes su teología y su cosmología en las contribuciones que ellas hacen a su visión del mundo. Pero esta consonancia (como muestra la historia) es una relación tentativa, constantemente bajo examen, en permanente y suave desplazamiento. (McMullin 1981, 51-52)

Esta es la propuesta que intentaré examinar.

\section{Análisis crítico del concepto de consonancia}

Si bien la idea de McMullin fue mayormente bien recibida, desde su primera formulación en 1981 ha tenido diversos vaivenes y retoques. Así no parece significar lo mismo el concepto de cooperación de Barbour (en sentido fuerte y débil) que las nociones de congruencia de Polkinghorne, convergencia y complementariedad de Karakash y Schäffer-Guignier y las de confirmación y contacto que propone Haught (Artigas 2000, 29-32; Papanicolau 2003, 13).

El término consonancia sugiere que hay áreas de correspondencia, conexión o relevancia entre los conocimientos de la naturaleza tal como los 
conciben las ciencias naturales y como los ha desarrollado la teología. A mi entender, se trata de subrayar la congruencia espontánea que resulta del ejercicio autónomo de diferentes disciplinas, como una señal de apoyo mutuo en el común sendero hacia la verdad. Puede decirse que este fenómeno se ha hecho particularmente visible desde el momento en que las nuevas teorías científicas introducen perspectivas de gran escala (sea en el dominio de la materia entendida como substrato universal, como en la física cuántica; sea en el dominio del Universo como totalidad, en el caso de la cosmología; sea en el terreno del dinamismo universal, según lo propone la teoría de la evolución) que se muestran en condiciones de una comparación casi directa con los planteos de la filosofía y la teología. Como lo ha señalado San Juan Pablo II, "la unidad que percibimos en la creación, sobre la base de nuestra fe en Jesucristo como Señor del universo, así como la correspondiente unidad por la que luchamos en nuestras comunidades humanas, parece reflejarse y reforzarse en los hallazgos reveladores de la ciencia contemporánea” (Juan Pablo II 1989, 20). Dada la índole de largo alcance de las teorías científicas actuales no sorprende que la consonancia aparezca también entre distintos ámbitos dentro de la misma ciencia. El mismo Juan Pablo II ha destacado este hecho a propósito de la visión evolutiva del universo, cuando sostenía que "la convergencia, de ningún modo buscada o provocada, de los resultados de trabajos realizados independientemente unos de otros, constituye de suyo un argumento significativo en favor de esta teoría.” (Juan Pablo II 1996, 5).

Ahora bien, esa consonancia (tomando el término, si se quiere momentáneamente, a manera descriptiva) puede entenderse, en un extremo, como mera coincidencia, y en el otro como signo de un acercamiento hacia un discurso común y una utópica súper unificación del conocimiento. Ante la contundencia del hecho, las reacciones no se han demorado, y pueden citarse ejemplos bastante cercanos a cada uno de esos extremos. A partir de la huella abierta por McMullin, varios estudiosos han procurado asumir este fenómeno cultural en términos más equilibrados, dando lugar a la idea de consonancia como propiedad natural del intercambio entre disciplinas (Steinke 2006, 57-59). 


\subsection{El diálogo desde la consonancia}

Según la tipología de Barbour, mayoritariamente aceptada, la consonancia correspondería al ámbito del diálogo. De modo que esta idea equidista tanto del mero paralelismo sin conexión como del intento de introducir argumentos tomados de una disciplina en la justificación de las afirmaciones de otra.

Hay que decir que, de acuerdo al panorama actual (Fulljames y Stolberg 2000), las otras variantes han perdido fuerza. El conflicto ciencia-fe tiende a descartarse porque supone en todos los casos algún error en la comprensión de una parte hacia la otra. La independencia es considerada insuficiente, pero a la vez actúa como contrapeso de un enfoque excesivamente integrador. Incluso en ciertos temas es vista como la mejor opción. Finalmente, el modelo de integración es en general deseable pero se dificulta considerablemente en razón de la pluralidad de visiones filosóficas y teológicas, como así también de las teorías en pugna dentro de la ciencia. Esta fragmentación se ha profundizado en las últimas décadas con el avance de las propuestas de inspiración posmoderna y posfundacionalista. Algunos han destacado también el contraste entre el sentido analógico y misterioso de las afirmaciones teológicas con la precisión y lógica explicativa de la ciencia. A ello se añade la diferencia fundamental entre el carácter progresivo y acumulativo del conocimiento científico y el desarrollo no lineal de la teología (Fulljames y Stolberg 2000, 36).

Los términos que se emplean en la caracterización del diálogo entre ciencia y teología, como los límites respecto de su defecto o exceso, son bastante lábiles. Un referente como John Haught apela a expresiones que suenan confusas cuando propone su división personal de la tipología siguiendo muy de cerca la de Barbour. En efecto, la categoría de contacto, que evoca la del diálogo, consiste en reconocer a la vez la distinción entre ciencia y religión y la existencia de "implicaciones" e "interacción” entre ambas (Artigas 2000, 31). Otros han introducido categorías intermedias entre el diálogo-consonancia y la integración, a saber, la asimilación (que supone la interacción efectiva y la acomodación entre las partes) y la correlación 
(una suerte de aproximación oscilante, según los temas, entre la consonancia y la asimilación) (Fulljames y Stolberg 2000).

Ahora bien, como en todo diálogo, es pertinente fijar las condiciones que lo hagan posible y fructífero. Siguiendo a Peters (2001, 228), las condiciones básicas serían:

1. Cada parte del diálogo debería tener una posición para proponer;

2. Cada parte del diálogo debería comenzar con una disposición hacia la apertura combinada con la voluntad de escuchar con simpatía la postura adelantada por los representantes de las otras tradiciones;

3. El diálogo genuino requiere una disposición de amor, tanto hacia el otro como hacia la verdad;

4. El diálogo genuino requiere suficiente tiempo y perseverancia para discutir asuntos en forma profunda y minuciosa.

Las cualidades subjetivas constituyen un dato insoslayable para asegurar la buena marcha del diálogo y sostener razonables expectativas de progreso. La actitud de acogida supone desde ya el respeto al espacio de manifestación del otro y el reconocimiento de la parte de verdad que, eventualmente, le pueda corresponder. Pero con eso no basta. Más bien hay que decir que "esta aceptación no debe ser sólo pasiva, en el sentido de que no neguemos las conclusiones, sino también activa, en el sentido de que integremos esas conclusiones con las que se deriven de nuestra propia disciplina." (Coyne y Omizzolo 2005, 161-162).

Por otra parte, la consonancia es un supuesto metodológico que afecta no solamente a la consistencia de la visión del mundo sino también de la teología y hasta de la ciencia. En otras palabras, desde la vocación de diálogo sustentada en la idea de consonancia se plantea la necesidad de discriminar criteriosamente entre las diferentes posturas teóricas que surgen de la labor científica y teológica, a fin de presentar al otro la visión más "confiable”:

By assuming that consonance exists between a theological view and a specific scientific theory, one implies that one should search for a suitable interpretation of the concepts involved. Probably such a search places both sets of concepts in a (not necessarily complete) metaphysical perspective. As this search does so, 
it is not only the world-view or the metaphysics that changes. The terms at the theological and scientific levels can also change meaning. Precisely what gives way and what remains unchanged depends on the relative importance of the various elements and on their reliability. In general, the science is considered to be the most reliable. In such cases, my proposal resembles an interpretation of the world as known through science: we reflect upon the knowledge provided by science and develop contemporary concepts to express our faith. (Drees 1990, 105)

Usualmente esa confiabilidad parece darse prioritariamente del lado de la ciencia, pero en ella también hay lugar para diferentes interpretaciones de sus teorías, sobre todo en los campos más avanzados o controversiales. Un caso referencial es el de la física cuántica. Otro ejemplo muy discutido es el del principio de inercia en relación con la acción conservadora de Dios (Pannenberg 1981). En estos y otros tópicos se pone de manifiesto la influencia que ejerce sobre la mentalidad del hombre de ciencia su propia visión del mundo y su perspectiva religiosa. Más allá de los problemas que supone la convalidación de confiabilidad respecto a los interlocutores, y dado que, aún bajo el criterio de tomar en cuenta "la mejor ciencia disponible", nos encontramos a veces con distintas aproximaciones teóricas, es necesario que la metafísica y la teología se ocupen de entrar en consonancia con cada una de ellas, a la manera en que lo han hecho con los distintos lenguajes y realidades culturales con las que han debido interactuar. De este modo se dará el primer paso hacia la verdad, que es el de la credibilidad.

Un asunto crucial en toda instancia de diálogo tiene que ver con la dimensión lingüística. En varios ambientes circula una versión alternativa de la idea de los “dos magisterios" de Stephen Gould, a saber, la teoría de los “dos lenguajes", el de la ciencia y el de la teología. Casi como evocando el episodio de Babel, quienes adhieren a esta postura consideran extremadamente limitadas las posibilidades de comunicación a partir de las divergencias de código que tienden a aislar a las disciplinas. Puede reconocerse en esta posición un acercamiento al análisis posmoderno de los relatos, siguiendo la línea abierta por François Lyotard. 
Además, aquí se tiene en cuenta una concepción epistemológica mucho más crítica respecto del método y las posibilidades de la ciencia, en clara superación del modelo neopositivista que prevalece entre los partidarios del conflicto o la independencia de dominios. Para entender el concepto de consonancia es preciso introducir elementos sociológicos y hermenéuticos (puede pensarse en ideas como "comunidad", ya referidas a grupos intelectuales o a sociedades de creyentes, como también en los consagrados "paradigmas" de Kuhn) importantes en la evaluación del conocimiento científico.

Algunos autores, como Willen Drees, tienen menos entusiasmo con respecto a los caminos de la consonancia. Según este investigador se trata de una idea útil, pero de suyo demasiado vaga, ya que la mera ausencia de contradicción o incluso la presencia de una cierta correspondencia entre las conclusiones teológicas y científicas dependen de una visión general del mundo cuya amplitud suele dar acogida a muy diversos puntos de vista. En otras palabras, la consonancia precisa de ciertos criterios para no confundirse con una mera construcción ad hoc.

Entre ellos, está el supuesto general del realismo crítico, ampliamente desarrollado en relación a esta temática, y con el que Drees está de acuerdo. Pero a su entender no debe pensárselo en forma ingenua, sino a partir de que tanto la teología como la ciencia tienen un aspecto de construcción que no puede ignorarse. Ni los registros "objetivos" de la observación empírica ni la rigidez de la norma sola Scriptura pueden soslayar la instancia hermenéutica y la intermediación de los lenguajes. Por eso la consonancia no es meramente descriptiva sino, al menos en parte, un modo de entender la realidad desde ciertas instancias de construcción. Por eso Drees habla de consonancia constructiva (1990, 105-107).

Igualmente se ha hablado de "disonancia” en relación a ciertas perspectivas científicas supuestamente incompatibles con la fe, como las que tienen que ver con el origen y destino final del universo. Este ultimo término fue incorporado por Robert Russell para señalar aquellos puntos de claro conflicto entre la perspectiva científica y la teológica, como es a su juicio la perspectiva catastrófica del final del Universo en contraste con la renovación 
universal anunciada por el cristianismo. Por su parte, Mc Fague considera disonante la pauta de la selección natural como mecanismo de evolución biológica con la consigna cristiana del amor y la protección privilegiados hacia el más débil (cit. por Edwards 1998, 380-381). Peters entiende que ciertos desarrollos de las neurociencias entran en consonancia con el rechazo de la teología cristiana respecto al dualismo mente-cuerpo, pero a la vez surgen disonancias en torno a la interpretación materialista del alma humana. Es preciso considerar cuidadosamente esos casos para no formular apresuradamente supuestas disonancias. Así se ha dicho, p.ej., que la inmensidad temporal del universo no afecta al concepto teológico de "plenitud de los tiempos en Cristo", ni la aventurada idea de una multitud de universos bastaría para conmover el carácter único del acontecimiento de la Encarnación (Poupard 1994,76). Igualmente, si la consonancia no basta para justificar afirmaciones científicas o teológicas, no se ve tampoco que la disonancia aporte un signo decisivo para rechazarlas.

\subsection{La consonancia hipotética}

La idea de consonancia según surge de los trabajos de McMullin tiene, según la opinión de otros estudiosos, un carácter optimista, comprometido o "fuerte" que debería ser matizado. Es así que Peters propone establecer una distinción entre "consonancia fuerte” y "consonancia débil”: "Consonancia en sentido fuerte significa acuerdo, armonía. Acuerdo o armonía podría ser un tesoro que tenemos la esperanza de encontrar, pero que todavía no hemos encontrado. Donde nos hallamos en este momento es trabajando con la consonancia en un sentido débil, esto es, por identificación de dominios comunes de interrogación.” (Peters 1996, 328). Las dificultades propias de los diferentes dominios epistemológicos, la falta de consenso sobre algunos temas críticos en materia de consonancia y la presencia de algunos contraejemplos (que, como ya he adelantado, se han denominado "disonancias") sugieren un camino más cauto. Se trata de plantear la consonancia ya no como un hecho definitivamente establecido sino como una cierta hipótesis de trabajo, como una aspiración que, en principio, tiene 
una razonable oportunidad de ser satisfecha. De ahí que se haya propuesto la denominación de consonancia hipotética:

Consonance evokes a sense of correspondence, resonance, harmony. "Hypothetical" places it in the form of a question, the form of an inquiry. Whether science and religion may discover consonance in their respective domains of knowing is the question to be pursued. Dialogue is the path for pursuing this question. According to the hypothetical consonance model, we begin with mutual respect between natural science and theological reflection. We presume that both science and theology are concerned with truth about the one reality in which we live and that, at least hypothetically, we ought to be able to find a common domain of discourse about it. Hypothetical consonance does not claim at the outset that science and religion have already converged; but it provides sufficient warrant to set an agenda of inquiry. Dialogue is the initial mode of inquiry, whereby we accept Gould's admonition to “keep talking. (Peters 2001, 228)

Al plantearse en estos términos atenuados, la consonancia resulta más flexible ante ciertos casos de disonancia (aparentemente) insanable. Sin embargo, en la medida en que se adopta un marco de aproximación basado en la consonancia hipotética, cabe esperar que las desavenencias se desvanezcan o al menos sean planteadas en términos menos conflictivos (Coyne y Omizzolo 2005, 162).

Además de las disonancias, el modelo hipotético permite absorber las dificultades de un solapamiento restringido. Quiere decir que la consonancia no debe esperarse en todos los temas, tal vez ni siquiera en la mayoría. En definitiva, no pueden ocultarse los contrastes entre el tipo de saber de la ciencia y la teología en términos de evolución histórica, perspectiva causal y pautas de validación. En efecto, y para no extendernos más allá del primer punto, resulta decisivo considerar en cualquier escenario de relación entre ciencia y teología la disparidad histórica que arrastran en su desarrollo ambas disciplinas. La teología cristiana ya estaba completamente consolidada en su estructura fundamental cuando se produjo la revolución científica copernicana. Por otra parte, el dinamismo y la creatividad de los hombres de ciencia en los últimos 300 años contrastan nítidamente con 
la relativa estabilidad de las propuestas teológicas en ese mismo período. Es evidente que ambos saberes marchan a ritmos distintos. El problema se ha agudizado en cierto modo a partir de las últimas teorías científicas, cuyo perfil y lenguaje las pone bastante lejos de los supuestos intuitivos de la metafísica realista tradicional a la que se ha amoldado la mentalidad occidental y que ha servido de soporte a la teología tradicional:

Many people have a very outdated picture of what science tells us about the world. They think of it as a clockwork world; the sort of universe that Laplace talked about at the end of the eighteenth century - his notorious statement that if a calculating demon knew everything that is happening now, that demon could predict the total future and retrodict the whole of the past. That certainly was the idea of physical process then, but it's not now. The universe is more open, more subtle, and more supple in its character than people in the eighteenth or nineteenth century could know. Unfortunately, this hasn't really permeated a great deal of theological thinking. People, and especially theologians, should try to familiarize themselves with scientific ideas. (Harris 2017)

De la misma manera, y dado el carácter hipotético de la ciencia (y hasta cierto punto de la teología) se corre el riesgo de establecer consonancias transitorias, o sea que una conexión ostensible entre las conclusiones de ambas disciplinas sobre una misma cuestión pueda desvanecerse posteriormente a causa de una corrección en esas mismas hipótesis. Por último, cabe evaluar el riesgo de una consonancia asimétrica, que actualmente parece acontecer, según la cual la instancia científica aparece con mayor peso propio y como un condicionante más enérgico que su par de la teología.

A mi juicio, lo que amerita un análisis más cuidadoso es la consonancia como regla heurística y como criterio selectivo entre teorías. Los casos ya constatados parecen alentar esa estrategia, si bien conviene insistir en que de ningún modo se lo tome como prueba sino, en términos de Santo Tomás, como un "signo leve" que dé lugar a la sospecha, que es el nivel anterior al de la hipótesis. Así como la teología "llegó antes” que la ciencia a establecer un tiempo cero del comienzo del mundo, ¿por qué la ciencia misma no podría llegar antes que la teología al descubrimiento de otra verdad? 
Bennett afirma que una metodología basada en la consonancia hipotética no está exenta de riesgos. En efecto, la mutua interacción a través de la crítica podría traducirse en una aprobación o desaprobación lisa y llana de las afirmaciones de la otra parte, y en definitiva en una suerte de "impureza" epistémica. Pero reivindica este encuadre en cuanto no se le pide que evite los desacuerdos, sino la autosuficiencia y la beligerancia.

Por mi parte, agregaría aquí que si la consonancia se toma como hipótesis, es natural tener en cuenta que pueda fallar. La cuestión es si la firmeza de este supuesto está en condiciones de habilitar un cinturón lakatosiano que la proteja. En tal sentido, el concordismo creacionista ha mostrado una frondosa imaginación para construir argumentos ad hoc.

Todas estas situaciones escabrosas no deberían disminuir la confianza en el modelo de consonancia hipotética. No debe perderse de vista que la principal fuente de justificación para cada saber estará siempre dentro de sí mismo, o en todo caso en conexión con los supuestos sapienciales. Allí donde la consonancia no funcione no debería recaer la sospecha, o en todo caso debería tomárselo como un indicador subjetivo, a mitad de camino entre una refutación propiamente dicha y una "corazonada".

\section{Estudio de casos}

Hoy es posible reconocer varios ejemplos locuaces de consonancia. Sin duda el más emblemático tiene que ver con las teorías cosmológicas referidas al origen del Universo y su cotejo con el relato bíblico y la idea teológica de creación. Además de su nitidez, el caso se potencia a partir de aquel controvertido discurso de Pío XII que, según una lectura algo superficial, parecía casi como una bendición del modelo del Big Bang. Me detendré un poco en él por estos motivos, para luego limitarme a una breve referencia sobre otros ejemplos.

Ante todo, la investigación histórica ha contribuido a relativizar la fuerza atribuida a las palabras del Pontífice. Estudios como los de Lambert y Tanzella-Nitti muestran que, leído en un contexto de ocasión que lo despoja de solemnidad y examinando de cerca sus expresiones, el discurso de Pío 
XII dista de ser tan categórico sobre lo que McMullin llamaría el respaldo de una disciplina hacia otra (Lambert 1997, Tanzella-Nitti 2017).

En segundo lugar, la hipótesis cosmológica de la existencia de un tiempo cero no debería relacionarse principalmente con las palabras bíblicas "En el principio", sino más bien con la condición contingente del universo, de la que se desprende la necesidad de un vínculo creador. Vale recordar que ha sido justamente el reconocimiento de esa contingencia, sobre todo en la famosa bula del Arzobispado de París de 1277, la que impulsó, a la larga, la legítima autonomía de la ciencia natural con respecto a la teología.

Tercero, la aceptación de esta consonancia ha tenido un fuerte impacto en la concepción de ciertas nociones que hasta hace poco parecían intuitivas y que, de alguna manera, son parte del lenguaje teológico: espacio, tiempo, materia y causalidad ya no pueden tomarse en un sentido que llamaríamos "local" o sesgado, y estos nuevos escenarios han suscitado a su vez nuevos lenguajes e interpretaciones en el ámbito de la teología de la creación, la cristología y la escatología.

Finalmente, no olvidemos que, debido a sus dificultades teóricas, la hipótesis de la singularidad inicial ha sido puesto bajo examen, y de hecho existen modelos como el de Stephen Hawking que dejan de lado el valor $\mathrm{t}=0$ por lo cual expresamente desactivan toda posibilidad de consonancia.

Otra de las cuestiones muy ventilada en los últimos años del siglo XX fue la del determinismo y la contingencia de las leyes naturales, principalmente a nivel cuántico, que estarían en conexión con el problema teológico de la Providencia y la libertad. En el contexto de lo que se tituló como "determinismo y acción divina” o también “el Dios de los agujeros”, se suscitó un debate interdisciplinario del que ha quedado una copiosa bibliografía.

También ha encontrado interés entre los científicos y los teólogos la cuestión de la irreversibilidad termodinámica del tiempo en conexión con la fe en una historia de la salvación que quiebra definitivamente el esquema antiguo del eterno retorno. Más recientemente se han insinuado otros vínculos por el estilo a partir de las investigaciones sobre teoría del caos y complejidad, queriendo establecer una consonancia a partir de la 
"sensibilidad de las condiciones iniciales" con el gesto amoroso de Dios en el comienzo de las cosas.

Permítanme dejar para el final la que es, a mi juicio, la más promisoria y cautivante de las consonancias, a saber, la que propone el denominado principio antrópico, complementado con el modelo evolutivo del universo. Fue propuesto en 1970 por el astrónomo Brandon Carter como un enunciado regulador en la determinación de las constantes cosmológicas. Este principio, que supone un contexto dominado por la perspectiva metafísica de tipo finalista, introduce fuertes resonancias en la temática teológica de la creación y el lugar del hombre en el cosmos como criatura escogida por Dios.

En todos estos casos las discusiones están en curso y tal vez pase mucho tiempo hasta que se aquieten. Pero cabe reconocer una tendencia muy provocadora en la dirección de un encuentro entre ciencia y teología que necesita de un análisis cauteloso y responsable.

\section{Necesidad de una mediación filosófica}

El tono fluido y amistoso en que se desenvuelve hoy el diálogo entre científicos y teólogos reafirma los progresos alcanzados y augura climas benignos para el entendimiento. Pero es justo reconocer que a menudo se descuidan ciertas prevenciones que exige todo intercambio, y llevados por el entusiasmo o la desaprensión los promotores de ese diálogo caen en conclusiones apresuradas o gestos demagógicos. No siempre las intenciones de una y otra parte han sido debidamente purificadas, ni se advierte el peligro del sincretismo o las soluciones precipitadas. En definitiva, se echa de menos la presencia de un marco de referencia que defina los parámetros fundamentales de la visión del mundo, de los distintos ámbitos epistémicos de abordaje y de los lenguajes comunes que puedan canalizar un diálogo genuino. Y todo ello es competencia de la sabiduría filosófica, desde su irrenunciable misión de ordenar y concertar las diferentes perspectivas del conocimiento. De ahí la necesidad de reivindicar el papel mediador de la filosofía para llevar a buen puerto las iniciativas de encuentro entre la ciencia y la teología. 
Varios autores me sirven de apoyo para esta convicción. En su Prefacio al notable libro de Artigas La mente del Universo, el Card. G. Poupard dice: "La ciencia está adquiriendo cada vez mayor conciencia de sus propios límites y de la necesidad de una fundamentación, pero, al mismo tiempo, desafía a la religión, pidiéndole más rigor intelectual en la presentación de su mensaje espiritual. Este reto es una llamada saludable a una mayor seriedad por parte de la religión. De este modo, ciencia y religión son llamadas a una seria reflexión filosófica y epistemológica, y a construir puentes sólidos que permitan una escucha y un enriquecimiento mutuos.” Más adelante agrega esta cita de Barbour: "Cualquier perspectiva sobre la relación entre ciencia y religión refleja supuestos filosóficos” (1997, 14 y 39). Por su parte el teólogo alemán Wolfhart Pannemberg, comentando un pasaje en el que San Juan Pablo II propone un diálogo entre la teología y la ciencia actuales a la manera de lo que ocurrió con Aristóteles en el Medioevo, sostiene que

la ciencia moderna no puede tomar simplemente el lugar que la filosofía aristotélica ocupó en relación con la teología medieval, como el Papa parece sugerir en un punto. Uno hasta puede dudar de que la ciencia moderna pueda reemplazar totalmente a la física aristotélica, y ni hablar de la metafísica y la lógica. La física moderna no reemplaza totalmente a la física aristotélica, si junto a la ciencia y a la teología una filosofía de la naturaleza permanece como una posibilidad. Los teólogos, por supuesto, pueden ser sus propios filósofos. (Papanicolau 2003, 10)

Aunque no puedo asegurarlo por tratarse de una cita indirecta, el texto evocado por Pannenberg es, muy probablemente, el siguiente:

No es propio de la teología incorporar indiferentemente cada nueva teoría filosófica o científica. Sin embargo, cuando estos descubrimientos llegan a formar parte de la cultura intelectual de la época, los teólogos deben entenderlos y contrastar su valor en orden a extraer del pensamiento cristiano alguna de las posibilidades aún no realizadas. El hilemorfismo de la filosofía natural de Aristóteles, por ejemplo, fue adoptado por los teólogos medievales, para servirse de él en el examen de la naturaleza de los sacramentos y la unión hipostática. Esto no significaba que la Iglesia juzgara la verdad o falsedad de la concepción 
aristotélica, ya que eso no es incumbencia suya. Significaba que ésta era una de las grandes concepciones ofrecidas por la cultura griega, que necesitaba ser comprendida, tomada en serio y contrastada en cuanto a su valor para iluminar diversas áreas de la teología. Los teólogos podrían preguntarse hoy si, con respecto a la ciencia, la filosofía y otras áreas del conocimiento humano contemporáneas, han llevado ellos a cabo este proceso extraordinariamente difícil, con la perfección con que lo hicieron estos maestros medievales. (Juan Pablo II 1989)

Lo que debe tenerse en cuenta, ante todo, es que ni la ciencia ni la teología son competentes para fijar las condiciones del diálogo en cuanto tal. En la práctica del diálogo interdisciplinario los representantes de las ciencias particulares aportan los contenidos cada vez más especializados de su saber y, en el mejor de los casos, una razonable especulación, de orden personal, sobre las proyecciones culturales de esa contribución. Ahora bien, desde el momento en que esto último cae fuera del objeto formal de su ciencia, queda al margen de la exigencia de rigor profesional que pone a un científico como interlocutor válido de la teología. Todo lo que ese científico pueda añadir a título de implicación de sus conocimientos no puede hacerlo en cuanto científico. Por su parte, la teología se acerca al diálogo con la ciencia como a algo que le es igualmente ajeno. Su “especialidad” es la Palabra de Dios, y los ecos que esa revelación pueda tener en el contexto de la visión científica del mundo exceden su competencia. Ante esta situación resulta indispensable dar el salto desde la interdisciplinariedad, que permanece en cierto modo bajo un esquema "horizontal", a la metadisciplinariedad, que bajo una suerte de "tercera dimensión" registra los distintos niveles de intelección y sus posibles vías de contacto. Hablando en particular sobre los intereses de la teología, el reciente documento de la Comisión Teológica Internacional afirma que "en esta crítica asimilación e integración de datos de otras ciencias por parte de la teología, la filosofía debe jugar un papel de intermediario. Pertenece a la filosofía, como sabiduría racional, insertar los resultados obtenidos por las variadas ciencias dentro de una visión más universal. El recurso a la filosofía en este papel mediador ayuda al teólogo a usar los datos científicos con la debida atención.” (Comisión Teológica Internacional $2012 \mathrm{n} .82$ ). 
La interdisciplinariedad entonces es un primer paso de acercamiento entre las ciencias, pero no alcanza para trascender hacia las cuestiones que desbordan ese plano y exigen respuestas más allá de los conceptos puramente disciplinares. Así la metadisciplinariedad aparece como complemento necesario de carácter sapiencial, fundamentalmente a partir de tres áreas: metodológica, temática y de fundamentación.

El trabajo de la filosofía exige una reflexión sobre las teorías científicas para alcanzar una justa valoración de su “peso ontológico”, pero también una cuidadosa comprensión del mensaje de fe, al que la teología debe ser capaz de presentar despojado de arcaísmos o prejuicios derivados de su encarnadura epocal. Como aclara Possenti, la ciencia se maneja con teorías y la teología con doctrinas. Y a la filosofía le corresponde el examen metafísico y antropológico que evalúe la posibilidad de conciliar las teorías con las doctrinas. La vocación metaempírica del intelecto hace posible introducir el discurso metafísico más allá del dato científico, lo cual contribuye además a recibir adecuadamente el aporte de la Revelación. Por contraposición, los conflictos entre religión y ciencia provienen de una interpretación filosófica equivocada del mensaje científico, impregnándolo de un trasfondo empirista, materialista y positivista (Possenti 2004). Como ejemplo, Artigas menciona la cuestión del reduccionismo:

El reduccionismo ontológico no se presenta como una consecuencia de la ciencia en sí misma, sino, más bien, como una idea 'metafísica' que, por tanto, debería discutirse utilizando no sólo datos científicos, sino también argumentos filosóficos. [...] el hecho mismo de que argumentamos acerca del reduccionismo muestra que existen dimensiones de la realidad que trascienden a la ciencia experimental: en efecto, cuando argumentamos acerca del reduccionismo, utilizamos argumentos que se refieren a la ciencia misma y, por tanto, no pertenecen al ámbito de la ciencia. (2000, 163-164)

\section{Supuestos de la consonancia}

Ya es tiempo de plantear la cuestión de los supuestos de la idea de consonancia. Aquel que nos sale al paso inmediatamente, y que ha sido destacado por 
encima de los otros, es sin duda el de la unidad de lo real. Aunque la teología y la ciencia aborden diferentes objetos formales, su punto de referencia está en el único mundo existente, que es el que nos rodea y que se muestra por igual a teólogos y científicos:

We live in one world and science and theology explore different aspects of it. The burden of our tale has been that, despite the obvious differences of subject matters, the two disciplines have in common the fact that they both involve corrigible attempts to understand experience. They are both concerned with exploring, and submitting to, the way things are. Because of this they are capable of interacting with each other: theology explaining the source of the rational order and structure which science both assumes and confirms in its investigations of the world; science by its study of creation setting conditions of consonance which must be satisfied by any account of the Creator and his activity. (Polkinghorne 2002)

Esto implica superar definitivamente la dicotomía entre "los asuntos del Cielo" y "los asuntos de la Tierra", como si la teología tuviese su mirada puesta exclusivamente en las alturas de lo sobrenatural, y dejase como menester de la ciencia profana el conocimiento de las cosas de este mundo. Lo cierto es que no solamente hay un sentido sobrenatural, que incumbe a la teología, en referencia al Universo visto como creación, como don y como sacramento de Dios, sino que también la ciencia puede contribuir a la reflexión teológica hacia una representación más adecuada de Dios, ya que todo lo que podemos atribuirle se toma por analogía del conocimiento de sus criaturas.

Justamente lo que otorga pleno sentido a la unidad de lo real es la universal dependencia con respecto a una Causa Primera. Sólo por referencia a Dios como Principio y Fin de todas las cosas es posible justificar esa unidad trascendental que pone a todo lo existente bajo la comunión del ser participado y de las leyes que lo gobiernan. Artigas lo resumen de esta manera: "deseo expresar que nuestro universo es racional, que funciona utilizando información, que le podemos atribuir una cierta creatividad, que hace posible la existencia de seres humanos que son racionales y creativos 
en sentido estricto, y que todo ello requiere un fundamento divino y una participación en la creatividad de Dios.” (Artigas 2000, 18).

Esta perspectiva trascendente de Dios contrasta con la ligereza de pensamiento que ha llevado a algunos científicos del presente a plantear una curiosa gnosis a partir de la búsqueda de la "Theory of Everything" (TOE) o también llamada "Great Unification Theory" (GUT). De acuerdo a esa perspectiva, detrás del régimen multifacético del Universo hay una clave extremadamente simple de la que depende toda razón, y que se suele identificar como "la mente de Dios". Tal vez buscando una consonancia más profunda, o una unificación final del conocimiento, o acaso un puente directo entre lo científico y lo religioso, se personaliza la ley cósmica y al mismo tiempo se banaliza la figura de Dios. El supuesto de la presencia divina como trasfondo y soporte de toda realidad no puede ciertamente reducirse a una fórmula o entidad platónica. Cualquier verdadero creyente sabe que el nombre más propio de Dios es Amor.

Cuando los científicos en su entusiasmo hablan de "teoría del Todo" y de la "mente de Dios" inevitablemente tratan de cuantificar lo que no es cuantificable: la intimidad, la bondad, la armonía, etc. [...] Habrá siempre una tensión entre la ciencia y la teología debido al carácter trascendental (más allá de la razón) de ésta. Pero en esta especie de búsqueda platónica de la "mente de Dios" en la nueva física, esa tensión podría ser fuente de un diálogo bastante creativo. Hoy, quizás más que en otros tiempos, la visión científica del mundo es el principal estímulo en la búsqueda de una visión más unificada y abre nuestras mentes a la vasta riqueza del universo de la cual ninguna disciplina puede apropiarse exclusivamente. La ciencia nos invita a esta visión y nos previene para no absolutizar los resultados científicos. Debemos cuidarnos de una peligrosa tentación de los cosmólogos en cuya cultura Dios es visto esencialmente, sino exclusivamente, como una explicación y no como una persona. Dios es la estructura matemática ideal, la teoría del todo. Dios es Mente. Un firme principio de la reflexión de la persona religiosa debe ser que Dios es más que eso y que su Revelación en el tiempo es más que una comunicación de información. Aún si descubrimos la "mente de Dios" no hemos necesariamente encontrado a Dios. La naturaleza de nuestra emergencia en un universo en evolución y nuestra incapacidad para comprenderlo, aun con todo lo que sabemos por la cosmología, puede ser 
una indicación de que en el universo Dios nos esté comunicando mucho más que información. A través de las limitaciones de las ciencias, podríamos ver el universo como una única revelación de Dios, la de que Dios es Amor. (Coyne y Omizzolo 2005, 165-166).

En segundo supuesto, que está estrechamente conectado con el de la unidad, es el que asume la inteligibilidad del universo, su capacidad para revelarse a la inteligencia humana, en cuanto es imagen y semejanza de su Autor. Este supuesto ha sido certeramente destacado por los epistemólogos actuales, sobre todo en relación a la posibilidad del conocimiento científico. Volviendo a Artigas, "la creencia en un orden natural subyacente y en la capacidad humana para conocerlo fueron dos factores que contribuyeron al desarrollo sistemático de la ciencia experimental moderna y continúan estimulando su progreso también en la actualidad. En la medida en que esas creencias han ayudado a conseguir algunos logros científicos y son anteriores a ellos, pueden ser consideradas como genuinos supuestos de la ciencia." (Artigas 2000, 57). Más aún, cuando el científico se persuade de la capacidad de su saber para entender el mundo, no puede sustraerse a la búsqueda de su fundamento. "Y esa búsqueda lo conduce hacia la teología”. (Steinke 2006, 37). La suposición es que hay un fundamento universal de nuestra comprensión y, como no puede contradecirse, la comprensión que tenemos de una disciplina debe complementar la de todas las demás. Se es más fiel a la propia disciplina -se trate de ciencias naturales, las ciencias sociales, la filosofía, la literatura o la teología- si se acepta este fundamento universal (Coyne y Omizzolo 2005, 161). Deseo agregar, por mi parte, que en la medida en que se enfatiza la dimensión constructiva de los distintos saberes y de la presunta consonancia entre ellos se da lugar a una reflexión, de suyo muy trabajada a lo largo del siglo XX, sobre la confluencia histórica entre cristianismo y ciencia. No parece desatinado pensar que el espíritu capaz de acoger y profundizar el mensaje de la fe, y que al mismo tiempo extrajo de su propia naturaleza una explicación científica del mundo, deba al fin y al cabo ser consonante consigo mismo.

Una variante de este supuesto que ha tomado cuerpo en los últimos años se refiere a la reposición del concepto analógico de racionalidad. Autores 
como Jean Ladrière han encontrado en él una clave fecunda para unificar el lenguaje interdisciplinario sin perder de vista el contenido analógico de la expresión. Según el filósofo francés, a lo largo de la historia se han incorporado diferentes manifestaciones del saber (filosófico, teológico, científico) y de la acción humana, que en todos los casos reivindican la autoridad de la razón. Más aún, todas esas manifestaciones evocan, de un modo más o menos explícito, el esquema de la analítica aristotélica según el cual todo saber supone alguna forma de justificación en base a principios. Por lo demás, la noción de racionalidad es suficientemente versátil para acoger procedimientos aparentemente tan lejanos entre sí como las pruebas empíricas de laboratorio y la exégesis bíblica, y no excluye bajo una misma denominación al cálculo matemático más abstracto y a la metáfora. (Ladrière 2003). En sintonía con esta propuesta, van Huyssteen afirma que "the hazy intersection between the diverse fields of theology and the other sciences is therefore not in the first place to be determined by exploring possible methodological parallels or degrees of consonance between theology and science. What should be explored first is a common and shared resource found in a richer notion of human rationality” $(1998,40)$.

En tercer lugar, corresponde introducir el supuesto del realismo, definido como la convicción según la cual nuestro conocimiento alcanza el verdadero ser de las cosas. No se trata de una presunción vacía o voluntarista, ni mucho menos de una pretensión agotadora de la realidad. Tampoco debe confundirse con la discusión acerca del realismo en la ciencia, que introduce problemáticas especiales como la referencialidad de los términos teóricos. Aquí se habla de un realismo que podría denominarse trascendente o fundamental, y que expresa la natural conformidad de nuestras facultades de conocimiento con aquello que es. De este realismo no pueden escapar ni los instrumentalistas (porque está asociado a una praxis apoyada en la realidad de los datos) ni los historicistas (porque el paradigma se convalida por resolución de problemas reales). Tampoco puede ser escamoteado por la teología, ya que el don de la fe interpela a una razón que es realista, y por eso enseña Santo Tomás que la fe no se aplica a un enunciado sino a la cosa. Desde luego, todos los autores están de acuerdo en que la realidad 
perseguida es esquiva y exige muchas veces retrocesos y replanteos, pero el espíritu del saber radica ante todo en la incondicional fidelidad al ser mismo de las cosas, como quiera que se nos manifieste.

Finalmente, el punto más delicado en este elenco de supuestos se refiere al equilibrio que ha de haber entre autonomía y complementariedad de las disciplinas. La justa independencia que debe preservarse en cada rama del saber constituye una conquista que hoy parece definitivamente afianzada. Detrás de ella corre la posibilidad de un diálogo promisorio que dé lugar a lo mejor que cada parte pueda poner de sí y desde sí. La consonancia no requiere que la ciencia incorpore "la noción del Brahman en sus ecuaciones. Los teólogos no necesitan trocar sus criterios de verdad por los que provienen de la ciencia. [Además] evita la ambición excesiva de presumir que ya sea la ciencia o la religión tienen cautiva la verdad. A menudo, la llamada guerra entre ciencia y religión es el resultado de los reclamos exclusivos del acceso a la verdad hechos por algunos reduccionistas o fundamentalistas." (Bennett y Peters 2002, xiv).

Ahora bien, esa autonomía debe conjugarse adecuadamente con el hecho de que ninguna de las disciplinas ostenta el dominio de la verdad completa y total. Más todavía, se reclaman entre sí para fortalecer su propio punto de vista. Con justas palabras de Polkinghorne, "la religión sin ciencia está confinada; no llega a abrirse completamente a la realidad. La ciencia sin religion está incomplete; lo llega a alcanzar lo más profundo de su capacidad de comprensión”. (Steinke 2006, 62). No obstante su acuerdo fundamental con McMullin en el texto que presentamos al comenzar la exposición de la idea de consonancia, el teólogo alemán es de la idea de que la consonancia, en cierto sentido, también implica un soporte o sustento racional. No en la línea de la justificación propiamente dicha, como si por ejemplo la doctrina de la creación dependiese estrictamente de una premisa científica, sino como una instancia que convalide su sentido y le permita ser formulada en el contexto de inteligibilidad apropiado.

If there were no consonance with the description of the reality of our world, then our faith in creation would become empty. That would endanger our faith in 
God. Our faith in God is essentially founded in the notion that He is the creator of everything, and that He has power over everything. God would not have this power if He were not the creator of everything. The consonance between science and theology, in some way, also supports the Christian affirmations - but not in the sense that theology should be modeled after scientific findings, efforts, and so on. Theology moves on its own level of method." [...] "Science cannot tell theology how to construct a doctrine of creation, but you can't construct a doctrine of creation without taking account of the age of the universe and the evolutionary character of cosmic history. (Harris 2017)

Claro está que la cooperación de que estamos hablando no puede plantearse en términos compulsivos. Coyne y Omizzolo proponen hablar de una "invitación”, término que satisface con creces las demandas naturales de cortesía, pero que parece insuficiente ante la necesidad de complemento que cada disciplina posee. Es importante enfatizar la palabra "invitación” para preservar la independencia epistemológica de las distintas disciplinas. Somos libres de aceptar o no la invitación, de permanecer firmes dentro de la propia disciplina y continuar buscando la respuesta sin contaminarse con posibles soluciones que surjan en otros campos. Pero, nos parece que la invitación es real y bien fundada y que se requieren, por lo tanto, serias razonas para rechazarla. (Coyne y Omizzolo 2005, 161).

Lo que acabamos de expresar en torno a los supuestos, que desde ya amerita una discusión mucho más profunda, confirma la importancia de la mediación filosófica. ¿De dónde tomar, si no, ideas tales como unidad ontológica, inteligibilidad, realismo, etc.? Este punto ha sido extensa y acertadamente discutido por Artigas en su obra La mente del universo, sobre todo al cotejarlo con la perspectiva de otros autores, como Barbour. La idea central es que sólo la reflexión mediadora de la filosofía puede tender un puente que haga posible la interacción entre dos mundos conceptuales tan heterogéneos y, sobre todo, que no incluyen el diálogo como una instancia metodológica esencial. Por eso, "el primer paso que conduce hacia esa unidad [entre ciencia y religión] es una reflexión filosófica más que un verdadero diálogo entre dos compañeros diferentes”. (Artigas 2000, 32). 


\section{Algunas consideraciones críticas}

Creo, ante todo, que debe aplaudirse la intención de promover la unidad del conocimiento, el entendimiento pacífico y constructivo entre las disciplinas y la posibilidad de abrir caminos de creatividad estimulante. Tal como lo he comprobado al estudiar la obra de Jacques Maritain, la causa de la unidad del saber, cualquiera sea la forma que deba adoptar, representa una meta impostergable para nuestra cultura, que avanza con impulso irrefrenable hacia horizontes de conocimiento y aplicación técnica que desafían la imaginación más audaz. Las responsabilidades que supone la administración de ese conocimiento, las pautas de poder que se desprender de él, la amenaza de una progresiva desigualdad en el capital intelectual de los distintos pueblos, el deterioro de la identidad religiosa sofocada por el avance científico, son diferentes escenarios que pueden presentarse en el corto plazo si no se buscan instancias de contrapeso para llegar a una cosmovisión verdaderamente integradora, en el sentido de salvaguardar las competencias propias de cada especialidad y, al mismo tiempo, custodiar las verdades y valores fundamentales que inspiran la existencia humana.

Además me parece laudable la propuesta de apuntar a una mediación filosófica que le devuelva a este saber su misión ordenadora. En modo particular, estimo la impronta realista que se pretende asignar a los fundamentos del diálogo, poniendo como centro de gravedad el ser y la verdad de las cosas, conforme a una metafísica que esté a la altura de la vocación trascendente de la inteligencia humana. Voces encumbradas en esta discusión han señalado con firmeza que la mejor garantía de una consonancia capaz de iluminar a los saberes y abrir caminos de interacción fecunda está en nuestra capacidad para reconocer lo que las cosas son, para subordinar nuestros enfoques y rivalidades a la unidad y el orden que gobiernan en el Universo, y sobre todo para reconocer la dignidad de la búsqueda del otro y ponernos a su lado comulgando no sólo en la verdad sino en el amor.

No obstante, advierto algunos puntos poco satisfactorios en esta propuesta de la consonancia, sobre todo a partir de lo que más específicamente se pone en juego desde la perspectiva de cada uno de los autores involu- 
crados. Ante todo, veo un serio defecto en la semántica que oscila entre el saber religioso (entendido como una síntesis de tradiciones, rituales y plegarias afines a una comunidad) y la teología propiamente dicha. A mi entender no pueden plantearse los mismos parámetros en un caso y otro. Las religiones son, por lo menos en sus expresiones más arraigadas, una manifestación de la vocación trascendente del hombre, capaces de inspirar la orientación de vastos espacios culturales y de movilizar el corazón de las personas hasta extremos intangibles desde lo natural. La teología, por su parte, tiene una meta más modesta: pretende, dentro de cada culto, derivar las conclusiones que, a partir de un cierto núcleo doctrinal, quedan a su disposición a partir de la aplicación de los conocimientos y las reglas de la razón. Tengo la impresión de que la idea de consonancia es más consistente con el diálogo entre ciencia y teología que cuando se plantea el intercambio con lo religioso, donde la densidad y resonancia del mensaje es mucho mayor y trasciende, hasta cierto punto, las categorías lógicas de la compatibilidad.

En segundo lugar, discrepo con una especie de "naturalismo" teológico, que procura asignarle a este saber un formato excesivamente acomodado a los esquemas de la ciencia natural. Desde luego que, según se ha insistido en todo momento, damos por descontado que la teología es una ciencia, y se somete a ciertos criterios de construcción y desarrollo que emanan del sujeto y actúan como modesto envase de la Palabra. Se ha discutido durante siglos el significado del carácter científico de la teología, y no soy un experto en ese tema. Pero experimento cierta perplejidad ante la intención de presentar el discurso teológico con modelos demasiado cercanos al de las ciencias empíricas, donde el teólogo debería manejar hipótesis de investigación, experiencias de corroboración, etc. Tal vez se deslice aquí un cierto despotismo epistemológico que persiste en presentar el paradigma del método científico a la manera del esquema hipotético deductivo como carta de ciudadanía para el diálogo entre las disciplinas. (Comisión Teológica Internacional 2012, n. 81).

Aunque, repito, no conozco el tema en profundidad, me atrevo a pensar que en muchos de esos planteos "naturalistas" se escapa un dato esencial del saber teológico, a saber, su dependencia intrínseca del dato revelado y la 
regulación especial que ejerce sobre ella el Magisterio de la Iglesia. La luz de la fe, bajo la cual el teólogo contempla los dogmas que son los principios de su saber, impregna toda la labor teológica y debería presentarse como una diferencia cualitativa insoslayable a la hora de dialogar. En otras palabras, quiero decir que la teología se presenta al diálogo en carácter de ciencia, pero es más que eso, o lo es de un modo muy especial. Y francamente no veo, en casi ningún caso, que se haya puesto de relieve esa diferencia. Al contrario, abundan los comentarios acerca de la situación desfavorable que atraviesa la teología al confrontar con disciplinas aparentemente mucho más sólidas que ella. Pareciera que, al haberse ocultado o directamente sustraído su impronta sobrenatural, la teología tiene que abandonar para siempre el lugar especial que le corresponde en el concierto de las ciencias. Si bien apenas lo he mencionado, creo que el tema de la jerarquía del saber ha entrado en una etapa de confusión que tendrá, a la larga, un elevado costo.

Menciono, en tercer lugar, algunas reservas sobre la manera en que se interpreta el término "realismo" en este contexto. Por momentos adquiere una significación empalidecida, donde el calificativo de "crítico" lo transfigura poniendo en primer lugar el aspecto de construcción o acuerdo que el de la referencia hacia lo real. Muchos autores entienden el realismo como una expresión de deseos, o como un ingrediente más en la constitución del objeto de conocimiento. Y así se corre el peligro de plantear la consonancia como fin y no como medio, o de diluir su fundamento en una especie de consenso sin referencia propia al ser de las cosas. En resumen, creo que en la mayoría de los casos la opción por el realismo queda a mitad de camino y esteriliza proporcionalmente los esfuerzos emprendidos en su nombre.

Por último, advierto en algunos casos una dependencia o injerencia excesiva entre los saberes, principalmente cuando se trata de complementar la propuesta teológica. Reconozco sinceramente que no llego a comprender el aporte efectivo y sustancial que puede hacer la visión actual de la ciencia en la asimilación de los misterios de la fe acerca del mundo. No veo que la teoría del Big Bang o de la evolución de las especies añada elementos fundamentales a la concepción del Dios creador, tal como la contempla la filosofía y la teología. Claro que no son cuestiones superfluas, y que el 
intercambio no debe desdeñarse. Pero sigo creyendo que el instrumento privilegiado para desarrollar las grandes líneas de la reflexión teológica está en la filosofía tradicional, y todo cuanto se quiera proponer más allá de eso debe darlo por supuesto. En todo caso, el hecho de que la cultura contemporánea se halle impregnada de ciertos enfoques teóricos de largo alcance promovidos desde la ciencia no los convierte, de suyo, en una versión actualizada de los preámbulos de fe. Expreso estos pensamientos con reserva y a la espera de una meditación más prolongada. Pero haciendo pie en ciertos principios que, en mi modesto parecer, me conducen a ellos.

Concluyo, pues, aseverando que la idea de consonancia, abierta a los matices y salvedades ya comentados, es una propuesta generosa en perspectivas que contribuye, sin duda alguna, a enriquecer la comprensión y la práctica del diálogo entre ciencia y teología. Apoyada en la seguridad de nuestro arraigo en el ser de las cosas y su inteligibilidad, ella se ofrece como garantía de un entendimiento cuyas formas deberán definirse con trazos sutiles y no siempre precisos. En todo caso, dependerá no sólo de la vocación de diálogo, debidamente preparada, de los científicos y los teólogos, sino también de la oportunidad que se brinde a la filosofía para interceder con su reflexión e iluminar los caminos.

\section{Bibliografía}

Artigas, Mariano. 2000. La mente del Universo. Pamplona: EUNSA.

Barzaghi, Amerigo y Corcó, Josep. 2015. “Ernan McMullin's Thought on Science and Theology: An Appreciation.” Open Theology 512-523.

Bennett, Gaymon y Peters, Ted. 2002. Ciencia y religión en diálogo. Un puente en construcción Puebla UPAEP.

Byrne, Peter. 1995. "Theology and Scientific Understanding." En Companion Encyclopedia of Theology, editada por Peter Byrne y Leslie Houlden, 449-450. London-New York: Routledge.

Comisión Teológica Internacional. 2012. La teología hoy. Perspectivas, principios y criterios. Buenos Aires: Ágape Libros.

Coyne, George y Omizzolo, Alessandro. 2005. Caminantes del Universo - astronomía y búsqueda humana de sentido. Buenos Aires: CIAFIC. 
Drees, Willem. 1990. “Theology and Cosmology Beyond the Big Bang Theory.” En Science and Religion: One World - Changing Perspectives on Reality, editado por Jan Fennema e Iain Paul 99-130. Dordrecht: Luver Academic Publishers.

Edwards, Denis. 1998. "Original Sin and Saving Grace in Evolutionary Context.” En Evolutionary and Molecular Biology - Scientific Perspectives on Divine Action, editado por Robert Russell, William Stoeger y Francisco Ayala, 377-392. Vatican City State Berkeley, California: Vatican Observatory Publications - Centre for Theology and the Natural Sciences.

Fulljames, Peter y Stolberg, Tonie. 2000. “Consonance, Assimilation or Correlation? Science and Religion Courses in Higher Education.” Science and Christian Belief 12/1: 35-46.

Harris, Lyndon. 2017. “Divine action: An interview with John Polkinghorne.” Consultado el 19 de julio. http://www.crosscurrents.org/polkinghorne.htm

Huyssteen, Wentzel van. 1998. "Postfoundationalism in Theology and Science: Beyond Conflict and Consonance.” En Rethinking Theology and Science: Six Models for the Current Dialogue, editado por Niels Gregersen y Wentzel van Huyssteen. Grand Rapids: Eerdmans.

Juan Pablo II. 1989. “Carta al Director del Observatorio Vaticano.” L'Osservatore Romano (edición española) 12 de febrero: 19-21.

Juan Pablo II. 1996. “Mensaje a la Academia Pontifica de Ciencias.” L'Osservatore Romano (edición española) 25 de octubre, 5.

Ladrière, Jean. 2003. “Science et théologie.” Revue Théologique de Laval 34: 3-26.

Lambert, Dominique. 1997. "Monseigneur Georges Lemaître et le débat entre la cosmogie et la foi." Revue Théologique de Louvain 28: 28-53.

McMullin, Ernan. 1981. “How Should Cosmology Relate to Theology?” En The Sciences and Theology in the Twentieth Century, editado por Arthur Peacocke, 17-52. Notre Dame: Univeristy of Notre Dame Press.

Pannenberg, Wolfhart. 1981. “Theological Questions to Scientists.” En The Sciences and Theology in the Twentieth Century, editado por Arthur Peacocke, 3-16. Notre Dame: Univeristy of Notre Dame Press.

Papanicolau, Jorge. 2003. “Religión y Ciencia en el pensamiento de Juan Pablo II: importancia y responsabilidad del diálogo para una cultura verdaderamente humana." Teología 82: 83-113.

Peters, Ted (ed.). 1998. Science and Theology. The New Consonance: Boulder CO: Westview Press.

Peters, Ted. 2001. “The Science-Religion Dialogue - An Ecumenical Catalyst?” Dialog: a Journal of Theology 40/3, 223-229.

Peters, Ted. 1996. “Theology and Science: Where Are We?” Zygon 31/2, 323-343. 
Polkinghorne, John. 1996. Scientists as Theologians. London.

Polkinghorne, John. 2002. “Reduccionism.” voz en Giuseppe Tanzella-Nitti y Alberto Strumia Dizionario Interdisciplinare di Scienza e Fede. Roma: Urbaniana Univ. Press y Città Nuova.

Possenti, Vittorio. 2004. “Aspetti del dialogo fra scienza e fede.” En XXIX Semana Tomista Buenos Aires. Consultado el 19 de julio de 2017. http://www.sta.org.ar/ cms/index.php/xxix-dialogo-entre-filosofia-teologia-y-ciencias

Poupard, P. 1994. Après Galilée. Science et foi: nouveau dialogue. Paris: Desclée de Brouwer.

Poupard, P. 2000. "Prefacio" a Artigas, Mariano La mente del Universo.

Steinke, Johannes Maria. 2006. Konsonanz von Naturwissenschaft und Theologie. Gottingen: V\&R.

Tanzella-Nitti, Giuseppe. 2017. "Il Papa e il Big Bang. Il caso Pio XII - Lemaître (1951-1952) a propósito del rapporto fra cosmologia e creazione.” Consultado el 19 de julio. www.disf.org. 